\title{
Numerical Modelling of the Acoustic Pressure Inside an Axisymmetric Lined Flow Duct
}

\author{
Mohamed TAKTAK ${ }^{(1)}$, Mohamed Ali MAJDOUB ${ }^{(1)}$, \\ Mabrouk BENTAHAR ${ }^{(2)}$, Mohamed HADDAR ${ }^{(1)}$ \\ (1) Unité de Mécanique, Modélisation et Productique (U2MP) \\ Département de Génie Mécanique, Ecole Nationale d'Ingénieurs de Sfax, Université de Sfax \\ BP 1173 - 3038, Sfax, Tunisie; e-mail: mohamed.taktak.tn@gmail.com \\ (2) Laboratoire Roberval UMR UTC-CNRS n 6253. Université de Technologie de Compiègne \\ BP 20529 F60205 Compiègne cedex, France
}

(received March 22, 2011; accepted March 13, 2012)

\begin{abstract}
Numerical methods are mostly used to predict the acoustic pressure inside duct systems. In this paper, the development of a numerical method based on the convected Helmholtz equation to compute the acoustic pressure inside an axisymmetric duct is presented. A validation of the proposed method was done by a comparison with the analytical formulation for simple cases of hard wall and lined ducts. The effect of the flow on the acoustic pressure inside these ducts was then evaluated by computing this field with different Mach numbers.
\end{abstract}

Keywords: lined duct, mean flow, finite element method, axisymmetric duct.

\section{Introduction}

To predict noises from compressors, aircraft engines, and ventilation systems which are generally composed of an acoustic source and rigid wall or lined wave guides, many theoretical and experimental works were developed to characterize the acoustic propagation and radiation of sound from ducts with geometry and impedance discontinuities. The objective of these works is the development of efficient tools which can be used in the design phase to optimize the lining, geometries, and forms to reduce the radiated noise. Works based on the analytical theory such as (BI et al., 2003; 2006), and (Leroux et al., 2003) presented the MultiModal Propagation Method (MMPM) based on expanding sound pressure and axial velocity into double infinite series using the rigid duct modal basis and the projection coefficients evolution along the duct axis. This method was used to evaluate the scattering matrix and the acoustic pressure inside the duct of axially and circumferentially non uniform lined ducts. MeIssNER (2010) proposed an analytical method based on a general acoustic impedance expression and on conditions of impedance continuity at duct section changes to model the plane wave motion in a duct. This method was tested with one, two and three discontinuities in the duct cross-section. It was validated by a comparison with numerical results calculated on the basis of the oscillator method with a finite difference algorithm. Owing to the limitations of the analytical methods, especially when the geometry and impedance repartition become complicated (section variation, complicated segmented liners...), the numerical methods were developed: WATson et al. (1996) presented a finite element propagation model based on the Galerkin technique to extract the acoustic impedance of the liner. This method is interpreted as an approximation of the continuous acoustic field seen as an assemblage of rectangular finite elements. LIN (1998) developed a numerical method based on a least-squares finite-element method to solve the two dimensional Helmholtz equation in rigid walls and lined ducts. The validation of his proposed method was made by comparison with the boundary element method. DYKAS et al. (2010) proposed a numerical method of modeling acoustic waves propagation based on the resolution of the non-linear Euler equations. The resolution is made with the use of a numerical scheme of third-order accuracy in space and time. The method was successfully tested and validated. The above methods present efficient tools to 
understand and control duct systems, but in industrial applications, the flow is present and has an important effect on the acoustic behavior of these systems. Hence, this parameter was incorporated in developed theoretical techniques. ElNADY (2004) presented a combined analytical collocation mode matching technique to evaluate the inside acoustic pressure field of a rigid-lined-rigid duct in the presence of a mean flow. Peat, Rathi (1995) proposed a finite element formulation for modeling the sound field in a duct lined by porous materials in the presence of a mean flow. WATson, Jones (2006) made a comparison between two impedance eduction techniques based, respectively, on the convected Helmholtz equation and the Euler equation in the presence of a mean flow. Due to the complexity of three-dimensional geometries and flows inside modern nacelles which present complicated factors, the two-dimensional modelling of duct systems in the presence of a mean flow remains limited, and new techniques were developed based on three-dimensional modelling taking into account the complicated flow. NARK et al. $(2003 ; 2005)$ developed a code taking into account the three-dimensional geometries and tested the method using a rectangular section by comparison with the analytical solution. LAN, BREAD (2005) presented a numerical modelling of the acoustic propagation inside a lined duct by using a parabolic approximation of the convected Helmholtz equation based on the Dougherty formulation proposed by DougherTy $(1997 ; 1999)$ to solve it with efficiency and with low computational costs in the case of complex three-dimensional geometries. A development of this method for different kinds of flow (mean, grazing) is presented and validated in (WATSON et al., 2005; JONES et al., 2005), and (NARK et al., 2006). LAPKA (2009) developed a numerical method to compute the Insertion Loss of spiral ducts. For this, a three dimensional model using the finite element method was used. The numerical results were validated by comparison with experiments. The results of (LAPKA, 2009) confirm that the use of a spiral duct has a great potential to attenuate noise in duct systems. In a previous work (TAKTAK et al., 2011) proposed a numerical method of sound propagation modelling in three-dimensional ducts in the presence of a flow. This method is based on a three-dimensional finite element formulation. The results are compared to an analytical solution and show the validity of numerical implementation. They also illustrate the flow effects on the sound propagation inside the duct. In the literature, other techniques are used to model the duct system like the time domain method which was developed by (ÖZYÖRÜK, LONG, 1996; Stanescu et al., 1999; Reichert, Biringen, 1997) and (SBARDELla et al., 2001) because of its lowcost computing power and the frequency-domain methods by (McAlPINe, Fisher, 2003) and (ÖZYÖRÜK et al., 2004) which are also used because they are much faster. Also, we note some works presenting optimization methods for duct systems such as the works of (Chiu, 2009) and (Chang, Chiu, 2010) to optimize multi-mufflers flow ducts using, respectively, the simulated annealing technique.

In this paper, we begin by presenting the studied problem and its governing equations in Sec. 2. The proposed numerical method to compute the acoustic pressure with a mean flow inside an axisymmetric lined duct is also presented in Sec. 2. In this later section, the numerical formulation and the finite element discretization are detailed. In Sec. 3, results of the proposed numerical method are validated by a comparison with the analytical results and discussed to evaluate the flow effect.

\section{Description of the physical problem}

The studied duct is cylindrical. Figure 1 presents its symmetric part. It does not present a sudden section change but an impedance discontinuity caused by the liner which is supposed to be locally reacting characterized by its acoustic impedance $Z$. $\Omega$ is the acoustic domain inside the duct. The edge of the studied duct is composed of four parts:

- The rigid wall duct part $\Gamma_{W D}$,

- The lined duct part $\Gamma_{L D}$,

- The left transversal boundary $\Gamma_{L}$,

- The right transversal boundary $\Gamma_{R}$.

$\Gamma_{W D}, \Gamma_{L D}, \Gamma_{L}$ and $\Gamma_{R}$ are characterized respectively by their normal vectors $\mathbf{n}_{W D}, \mathbf{n}_{L D}, \mathbf{n}_{L}$ and $\mathbf{n}_{R}$. A uniform flow is present in this duct modeled by the vector $\mathbf{M}_{0}$ defined as:

$$
\mathbf{M}_{0}=\left(\frac{U_{0}}{c}\right) \mathbf{z}=M_{0} \mathbf{z}
$$

$M_{0}$ is the Mach number, $U_{0}$ is the flow velocity, and $c$ is the sound velocity.

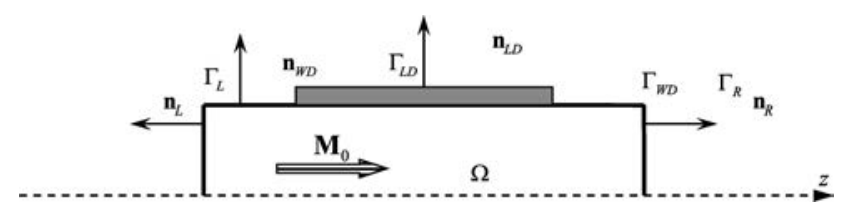

Fig. 1. Schematic of the studied problem.

\subsection{Computation of the acoustic pressure field within the duct}

\subsubsection{Governing equations}

To compute the acoustic pressure filed inside the studied duct, a modal pressure is imposed on the left boundary of the duct $\Gamma_{L}$ in the direction of increas$\operatorname{ing} z$, and a boundary condition is applied on the right 
boundary $\Gamma_{R}$ to eliminate the reflection phenomenon inside the duct, as presented in Fig. 2. The acoustic pressure inside the duct $p$ is the solution of the system containing the convected Helmholtz equation with boundaries conditions at $\Gamma_{W D}, \Gamma_{L D}, \Gamma_{L}$, and $\Gamma_{R}$ presented as follows (REDDY, 1993):

$$
\begin{aligned}
\Delta p+k^{2} p & +\frac{2 i \omega}{c} \cdot\left(\mathbf{M}_{0} \cdot \nabla p\right) \\
& -\mathbf{M}_{0} \cdot \nabla\left(\mathbf{M}_{0} \cdot \nabla p\right)=0 \\
Z \frac{\partial p}{\partial n_{L D}} & =\frac{\rho_{0}}{i \omega}\left(-i \omega+U_{0} \frac{\partial}{\partial z}\right)^{2}(p) \\
\frac{\partial p}{\partial n_{W D}} & =0 \\
p & =P_{m n}^{+} \\
\frac{\partial p}{\partial n_{R}} & =i k_{m n}^{+} p
\end{aligned}
$$

$\Delta$ is the Laplacien operator, $k$ is the total wave number, $\rho_{0}$ is the mass per volume unit and $\omega$ is the pulsation. $\nabla=\langle\partial / \partial r i m / r \partial / \partial z\rangle^{\mathrm{T}}$ is the modified gradient for axisymmetric problems with $m$ is the azimuthal wave number. $P_{m n}^{+}=J_{m}\left(\frac{\chi_{m n}}{a} r\right) e^{i k_{m n}^{+} z}$ is the imposed acoustic mode propagating in the direction of increasing $z$ with $n$ is the angular wave number. $\chi_{m n}$ is the $n$-th root satisfying the radial hard-boundary condition on the wall of the main duct and $a$ is the duct radius. $k_{m n}^{ \pm}$are the axial wave number associated to the $(m, n)$ mode and defined as:

$$
k_{m n}^{ \pm}=\frac{-M_{0} k \pm \sqrt{k^{2}-\left(1-M_{0}^{2}\right) k_{t}^{2}}}{\left(1-M_{0}^{2}\right)},
$$

$k_{t}$ is the transverse wave number. The sign + means that the axial wave number is calculated in the same direction as the flow while the sign - means that the axial wave number is calculated in the opposite direction of the flow.

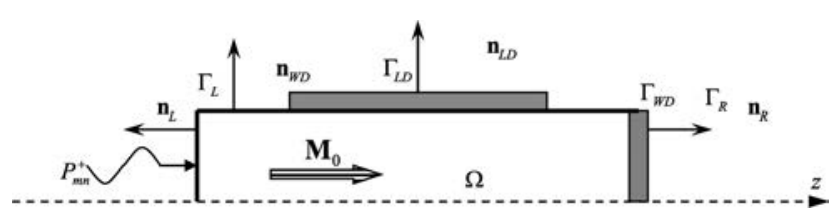

Fig. 2. Schematic of the theoretical model for the computation of inside acoustic pressure field.

\subsubsection{Variational formulation}

To solve the problem (2), the finite element method is used. The weak variational formulation of this problem is written as follows (REDDY, 1993):

$$
\begin{array}{r}
\Pi=\int_{\Omega}-(\nabla q \cdot \nabla p) r \mathrm{~d} \Omega+\frac{1}{c^{2}} \int_{\Omega}\left(\left(i \omega q+\mathbf{U}_{0} \cdot \nabla q\right)\right. \\
\left.\cdot\left(-i \omega p+\mathbf{U}_{0} \cdot \nabla p\right)\right) r \mathrm{~d} \Omega+\int_{\cup \Gamma_{i}}\left(q \frac{\partial p}{\partial n_{i}}-\frac{1}{c^{2}} \mathbf{U}_{0} \cdot \mathbf{n}_{i}\right. \\
\left.\cdot q\left(-i \omega+U_{0} \frac{\partial}{\partial n_{i}}\right)(p)\right) r \mathrm{~d} \Gamma_{i}=0,
\end{array}
$$

$p$ and $q$ are respectively the acoustic pressure in the duct and the test function, $\mathrm{d} \Omega=\mathrm{d} r \mathrm{~d} z$ is the surface element. $\cup \Gamma_{i}$ presents the whole boundaries $(i=$ $L D, L, R)$. The third integral includes boundaries conditions. This integral is composed of three parts:

- Lined part $\Gamma_{L D}$ :

$$
\begin{array}{r}
\int_{\Gamma_{L D}}\left(q \frac{\partial p}{\partial n_{L D}}-\frac{1}{c^{2}} \mathbf{U}_{0} \cdot \mathbf{n}_{L D}\right. \\
\left.\cdot q\left(-i \omega+U_{0} \frac{\partial}{\partial n_{L D}}\right)(p)\right) r \mathrm{~d} \Gamma_{L D} \\
=-\rho_{0} \omega^{2} \int_{\Gamma_{L D}} q \frac{p}{i \omega Z} r \mathrm{~d} \Gamma_{L D} \\
-2 i \omega \rho_{0} U_{0} \int_{\Gamma_{L D}} q \frac{\partial}{\partial z}\left(\frac{p}{i \omega Z}\right) r \mathrm{~d} \Gamma_{L D} \\
-\rho_{0} U_{0}^{2} \int_{\Gamma_{L D}} \frac{\partial q}{\partial z} \frac{\partial}{\partial z}\left(\frac{p}{i \omega Z}\right) r \mathrm{~d} \Gamma_{L D} \\
+\rho_{0} U_{0}^{2}\left[r q \frac{\partial}{\partial z}\left(\frac{p}{i \omega Z}\right)\right]_{z_{L D 1}}^{z_{L D 2}}
\end{array}
$$

with $z_{L D 1}$ and $z_{L D 2}$ being respectively the beginning and the end axial coordinate of the lined part of the duct.

- The left boundary $\Gamma_{L}$ :

$$
\begin{gathered}
\int_{\Gamma_{L}}\left(q \frac{\partial p}{\partial n_{L}}-\frac{1}{c^{2}} \mathbf{U}_{0} \cdot \mathbf{n}_{L} \cdot q\left(-i \omega+U_{0} \frac{\partial}{\partial n_{L}}\right)(p)\right) r \mathrm{~d} \Gamma_{L} \\
=\left(\left(1+M_{0}^{2}\right) \cdot i k_{m n}^{+}-\frac{i \omega U_{0}}{c^{2}}\right) \int_{\Gamma_{L}} q r \mathrm{~d} \Gamma_{L} \cdot P_{m n} .
\end{gathered}
$$

- The right boundary $\Gamma_{R}$ :

$$
\begin{gathered}
\int_{\Gamma_{R}}\left(q \frac{\partial p}{\partial n_{R}}-\frac{1}{c^{2}} \mathbf{U}_{0} \cdot \mathbf{n}_{R} \cdot q\left(-i \omega+U_{0} \frac{\partial}{\partial n_{R}}\right)(p)\right) r \mathrm{~d} \Gamma_{R} \\
=\left(\left(1-M_{0}^{2}\right) \cdot i k_{m n}^{+}+\frac{i \omega U_{0}}{c^{2}}\right) \int_{\Gamma_{R}} q \cdot p r \mathrm{~d} \Gamma_{R} .
\end{gathered}
$$

\subsubsection{Finite element discretization}

To solve the proposed problem, the domain $(\Omega)$ is discretized with triangular finite elements, while edges 
are meshed by two-node finite elements, as presented in Fig. 3. Computation of the integrals of Eq. (4) is made by summing over the finite elements number of the elementary integrals (DHATT, Touzot, 1989):

$$
\begin{aligned}
I_{e 1}= & \int_{\Omega_{e}}-(\nabla q \cdot \nabla p) r d \Omega_{e}+\frac{1}{c^{2}} \int_{\Omega_{e}}\left(i \omega q+\mathbf{U}_{0} \cdot \nabla q\right) \\
& \cdot\left(-i \omega p+\mathbf{U}_{0} \cdot \nabla p\right) r \mathrm{~d} \Omega_{e}, \\
I_{e 2}= & -\rho_{0} \omega^{2} \int_{\Gamma_{e}} q \frac{p}{i \omega Z} r \mathrm{~d} \Gamma_{e} \\
& -2 i \omega \rho_{0} M_{0} \int_{\Gamma_{e}} q \frac{\partial}{\partial z}\left(\frac{p}{i \omega Z}\right) r \mathrm{~d} \Gamma_{e} \\
& -\rho_{0} M_{0}^{2} \int_{\Gamma_{e}} \frac{\partial q}{\partial z} \frac{\partial}{\partial z}\left(\frac{p}{i \omega Z}\right) r \mathrm{~d} \Gamma_{e},
\end{aligned}
$$

$I_{e 3}=\rho_{0} M_{0}^{2}\left[r q \frac{\partial}{\partial z}\left(\frac{p}{i \omega Z}\right)\right]_{z_{L D 1}}^{z_{L D 2}}$

$I_{e 4}=\left(\left(1+M_{0}^{2}\right) \cdot i k_{m n}^{+}-\frac{i \omega M_{0}}{c^{2}}\right) \int_{\Gamma_{e}} q r \mathrm{~d} \Gamma_{e} \cdot P_{m n}$,

$I_{e 5}=\left(\left(1-M_{0}^{2}\right) \cdot i k_{m n}^{+}+\frac{i \omega M_{0}}{c^{2}}\right) \int_{\Gamma_{e}} q \cdot p r \mathrm{~d} \Gamma_{e}$,

where $\Omega_{e}$ and $\Gamma_{e}$ are respectively the elementary triangular and two-node finite elements.

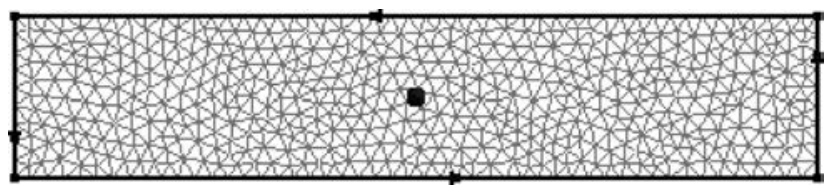

Fig. 3. The finite elements mesh of the studied duct element.

2.1.3.1. Elementary computation of the triangular finite element. For the triangular finite element composed of three nodes (1,2 and 3), the integral $I_{e 1}$ is written as follows:

$$
\begin{aligned}
& I_{e 1}=\left\langle\begin{array}{lll}
q_{1} & q_{2} & q_{3}
\end{array}\right\rangle \mathbf{K}_{e 1}\left\langle\begin{array}{lll}
p_{1} & p_{2} & p_{3}
\end{array}\right\rangle^{\mathrm{T}}, \\
& {\left[K_{e}\right]_{1}=\int_{\Omega_{\mathrm{ref}}}-\left(\nabla q \cdot \nabla p^{\mathrm{T}}\right) \operatorname{det} \mathbf{j} r \mathrm{~d} \xi \mathrm{d} \eta} \\
& +\int_{\Omega_{\mathrm{ref}}}\left(i \frac{\omega}{c}\left\{\begin{array}{c}
N_{1}^{\prime} \\
N_{2}^{\prime} \\
N_{3}^{\prime}
\end{array}\right\}+\mathbf{U}_{0} \cdot \nabla q\right) \\
& \cdot\left(-i \frac{\omega}{c}\left\langle N_{1}^{\prime} N_{2}^{\prime} N_{3}^{\prime}\right\rangle+\mathbf{U}_{0} \cdot \nabla p\right) \operatorname{det} \mathbf{j} r \mathrm{~d} \xi \mathrm{d} \eta,
\end{aligned}
$$

$\boldsymbol{j}$ is the inverse matrix of the Jacobien matrix $\boldsymbol{J}$ of the transformation from the reference element to the real base. $N_{1}^{\prime}(\xi, \eta), N_{2}^{\prime}(\xi, \eta)$ and $N_{3}^{\prime}(\xi, \eta)$ are the interpolation functions of the triangular finite element (DHATT, Touzot, 1989) defined by:

$$
\begin{aligned}
& N_{1}^{\prime}(\xi, \eta)=1-\xi-\eta, \\
& N_{2}^{\prime}(\xi, \eta)=\xi, \\
& N_{3}^{\prime}(\xi, \eta)=\eta .
\end{aligned}
$$

2.1.3.2. Elementary computations of the two-node finite element. For a two-node finite element belonging to the lined part of the duct composed of two nodes (1 and 2), $I_{e 2}$ and $I_{e 3}$ are computed as follows:

$$
\begin{aligned}
& I_{e 2}=\left\langle\begin{array}{ll}
q_{1} & q_{2}
\end{array} \mathbf{K}_{e 2}\left\{\begin{array}{l}
p_{1} \\
p_{2}
\end{array}\right\},\right. \\
& \mathbf{K}_{e 2}=\mathbf{K}_{e 21}+\mathbf{K}_{e 22}+\mathbf{K}_{e 23} \text {, } \\
& \mathbf{K}_{e 21}=\rho_{0} i \omega \int_{-1}^{1}\left\{\begin{array}{l}
N_{1} \\
N_{2}
\end{array}\right\}\left\langle\begin{array}{ll}
N_{1} & \left.N_{2}\right\rangle
\end{array}\right. \\
& \cdot \frac{\left\langle N_{1} N_{2}\right\rangle}{\left\langle\begin{array}{ll}
Z_{1} & \left.Z_{2}\right\rangle
\end{array}\left\{\begin{array}{l}
N_{1} \\
N_{2}
\end{array}\right\}\right.} \cdot \frac{L_{e}}{2} r \mathrm{~d} \xi, \\
& \mathbf{K}_{e 22}=-2 \rho_{0} U_{0} \int_{-1}^{1}\left\{\begin{array}{l}
N_{1} \\
N_{2}
\end{array}\right\}\left(\frac{\frac{2}{L_{e}}\left\langle-\frac{1}{2} \frac{1}{2}\right\rangle}{\left\langle Z_{1} Z_{2}\right\rangle\left\{\begin{array}{l}
N_{1} \\
N_{2}
\end{array}\right\}}\right.
\end{aligned}
$$

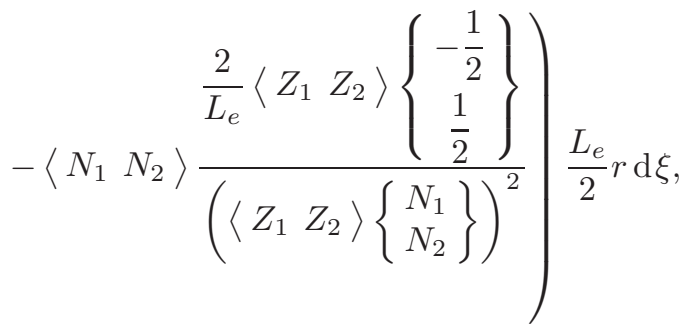

$$
\begin{aligned}
& \mathbf{K}_{e 23}=\frac{\rho_{0} U_{0}^{2}}{i \omega} \int_{-1}^{1} \frac{2}{L_{e}}\left\{\begin{array}{c}
-\frac{1}{2} \\
\frac{1}{2}
\end{array}\right\}\left(\frac{\frac{2}{L_{e}}\left\langle-\frac{1}{2} \frac{1}{2}\right\rangle}{\left\langle Z_{1} Z_{2}\right\rangle\left\{\begin{array}{l}
N_{1} \\
N_{2}
\end{array}\right\}}\right.
\end{aligned}
$$

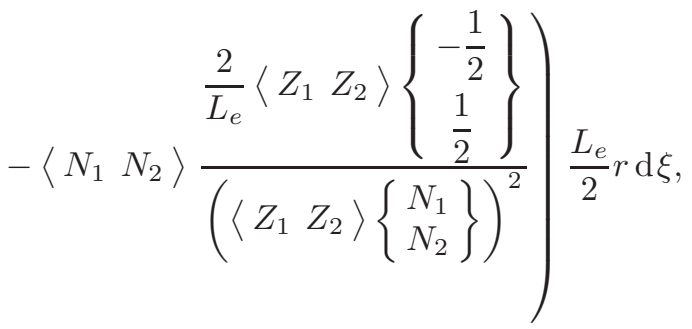

$Z_{1}$ and $Z_{2}$ are the acoustic impedance of each node of the two-node finite element; $L_{e}$ is the two-node fi- 
nite element length. $N_{1}(\xi)$ and $N_{2}(\xi)$ are the interpolation functions of the two-node finite element defined by (Dhatт, Touzot, 1989):

$$
\begin{aligned}
N_{1}(\xi, \eta) & =(1-\xi) / 2, \\
N_{2}(\xi) & =(1+\xi) / 2 .
\end{aligned}
$$

Computation of $I_{e 3}$ is done for the two-node finite elements on the lined part extremities.

$$
\begin{aligned}
& I_{e 3}=\left\langle\begin{array}{ll}
q_{1} & q_{2}
\end{array} \mathbf{K}_{e 3 Z 2}\left\{\begin{array}{l}
p_{1} \\
p_{2}
\end{array}\right\}-\left\langle\begin{array}{ll}
q_{1} & q_{2}
\end{array} \mathbf{K}_{e 3 Z 1}\left\{\begin{array}{l}
p_{1} \\
p_{2}
\end{array}\right\},\right.\right. \\
& \mathbf{K}_{e 3 Z 2}=\frac{\rho_{0} U_{0}^{2}}{i \omega} \cdot \frac{2}{L_{e}}\left\{\begin{array}{l}
0 \\
1
\end{array}\right\}\left(\frac{\left\langle-\frac{1}{2} \frac{1}{2}\right\rangle}{\left\langle Z_{1} Z_{2}\right\rangle\left\{\begin{array}{l}
0 \\
1
\end{array}\right\}}\right.
\end{aligned}
$$

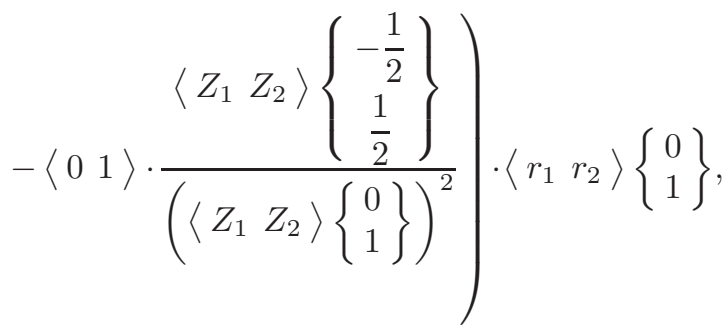

$$
\begin{aligned}
& \mathbf{K}_{e 3 Z 1}=\frac{\rho_{0} U_{0}^{2}}{i \omega} \cdot \frac{2}{L_{e}}\left\{\begin{array}{l}
1 \\
0
\end{array}\right\}\left(\frac{\left\langle-\frac{1}{2} \frac{1}{2}\right\rangle}{\left\langle Z_{1} Z_{2}\right\rangle\left\{\begin{array}{l}
1 \\
0
\end{array}\right\}}\right.
\end{aligned}
$$

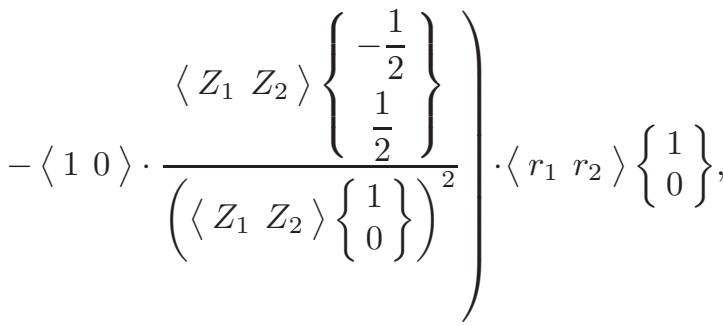

$r_{1}$ and $r_{2}$ are the radiuses of each corresponding real node.

Computation of the integral $I_{e 4}$ of a two-node finite element belonging to the left boundary is made as follows:

$$
\begin{aligned}
I_{e 4}= & \left\langle q_{1} q_{2}\right\rangle \cdot\left\{\begin{array}{l}
P_{1 m n} \\
P_{2 m n}
\end{array}\right\}, \\
P_{j m n}= & \left(\left(1+M_{0}^{2}\right) \cdot i k_{m n}^{+}-\frac{i \omega U_{0}}{c^{2}}\right) \\
& \cdot P_{m n} \cdot \int_{-1}^{1} N_{j}(\xi) \frac{L_{e}}{2} r \mathrm{~d} \xi, \quad j=1,2 .
\end{aligned}
$$

Computation of the integral $I_{e 5}$ of a two-node finite element belonging to the right boundary is:

$$
\begin{aligned}
I_{e 5}=\left\langle q_{1}\right. & \left.q_{2}\right\rangle \cdot \mathbf{K}_{e 5}\left\{\begin{array}{l}
p_{1} \\
p_{2}
\end{array}\right\} \\
\mathbf{K}_{e 5}= & \left(\left(1-M_{z}^{2}\right) \cdot i k_{m n}^{+}+\frac{i \omega U_{0}}{c^{2}}\right) \\
& \cdot \int_{-1}^{1}\left\{\begin{array}{l}
N_{1} \\
N_{2}
\end{array}\right\}\left\langle N_{1} N_{2}\right\rangle \frac{L_{e}}{2} r \mathrm{~d} \xi .
\end{aligned}
$$

Integration of the above integrals is made using the numerical Gauss integration method (DнAтT, Touzot, 1989). The assembly of different elementary integrals computed before is obtained as follows:

$$
\begin{aligned}
\mathbf{K}_{1} & =\sum_{1}^{N e l T} \mathbf{K}_{e 1}, \\
\mathbf{K}_{2,3} & =\sum_{1}^{N e l L D}\left(\mathbf{K}_{e 2}\right)+\mathbf{K}_{e 3 Z 1}+\mathbf{K}_{e 3 Z 2}, \\
\mathbf{K}_{5} & =\sum_{1}^{N e l R} \mathbf{K}_{e 5}
\end{aligned}
$$

with NelT being the number of triangular finite elements, NelLD being the number of two-node finite elements along the lined part, and NelR being the number of two-node finite elements at the right boundary of the duct. To solve the variational formulation (4), the matrices are arranged to obtain the following system:

$$
\begin{aligned}
\left\langle q_{1} \ldots q_{M}\right\rangle_{M} \mathbf{K}_{M \times M}\left\{\begin{array}{c}
p_{1} \\
\vdots \\
p_{M}
\end{array}\right\} & \\
& =\left\langle q_{1} \ldots q_{M}\right\rangle_{M}\{F\}_{M}, \\
\mathbf{K} & =\mathbf{K}_{1}+\mathbf{K}_{2,3}+\mathbf{K}_{5}, \\
\{F\} & =\left\{P_{j m n}\right\}_{\text {NnodeL }},
\end{aligned}
$$

$M$ is the number of nodes and NnodeL is the number of nodes at the left boundary. The resolution of this system allowed determination of the acoustic pressure in each node within the studied duct.

\section{Numerical results}

To validate the proposed finite element method, two cases of ducts were studied: a rigid wall and lined ducts. The chosen example consists in applying a modal pressure $P_{m n}^{+}$on the left boundary $\Gamma_{L}$ of a cylindrical duct. The geometric characteristics of the studied duct are: the radius $a=0.1 \mathrm{~m}$ and the length $L=0.5 \mathrm{~m}$. On the right boundary of the studied duct, an acoustic condition is applied to eliminate the reflection effects. The mesh of the studied duct is done 
by using 1128 finite elements. Afterwards, we compare the acoustic pressure fields obtained with the present numerical method and by an analytical formulation (LEsueur, 1988). The acoustic pressure is a complex value, hence, the real and the imaginary parts of the numerical and analytical values of the pressure are presented.

\subsection{Case No. 1: Cylindrical rigid wall duct}

The $(0,0)$ mode is imposed to the studied wall duct. Figures 4, 5, and 6 present a comparison between the analytical and numerical solutions of the acoustic pressure fields inside the duct at the frequency $f=1000 \mathrm{~Hz}$, respectively, for $M_{0}=-0.2,0$ and 0.2 .

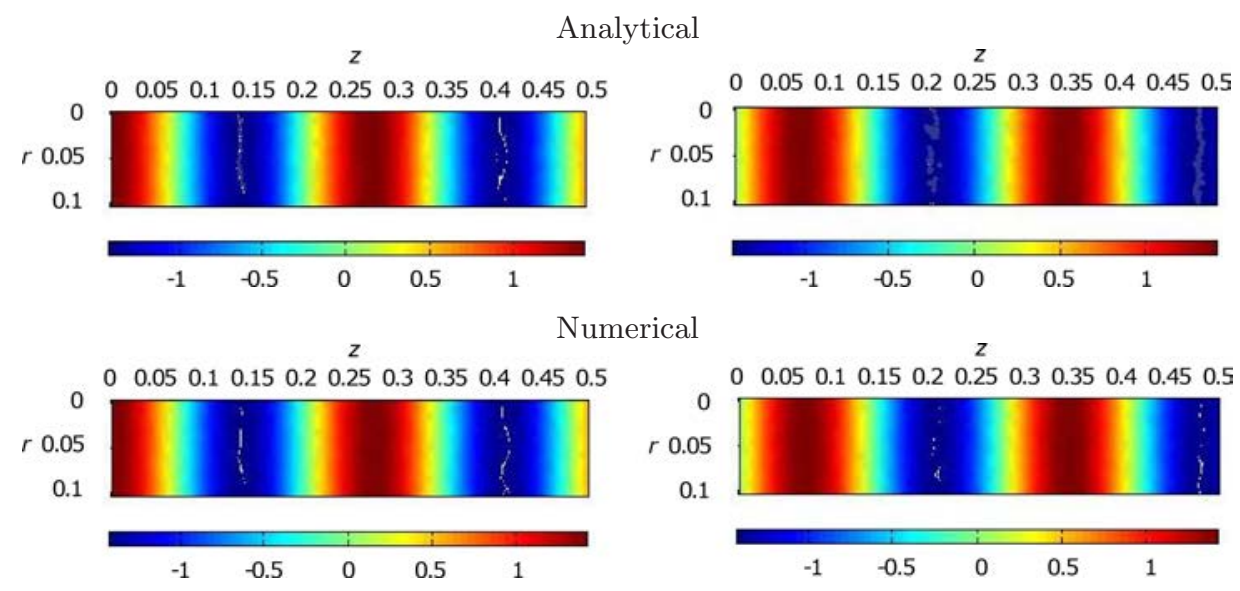

Fig. 4. Real part (left) and imaginary part (right) of the acoustic pressure in Pa inside of the studied wall duct at $f=1000 \mathrm{~Hz}$ and for $M_{0}=-0.2$ when imposing the $(0,0)$ mode.

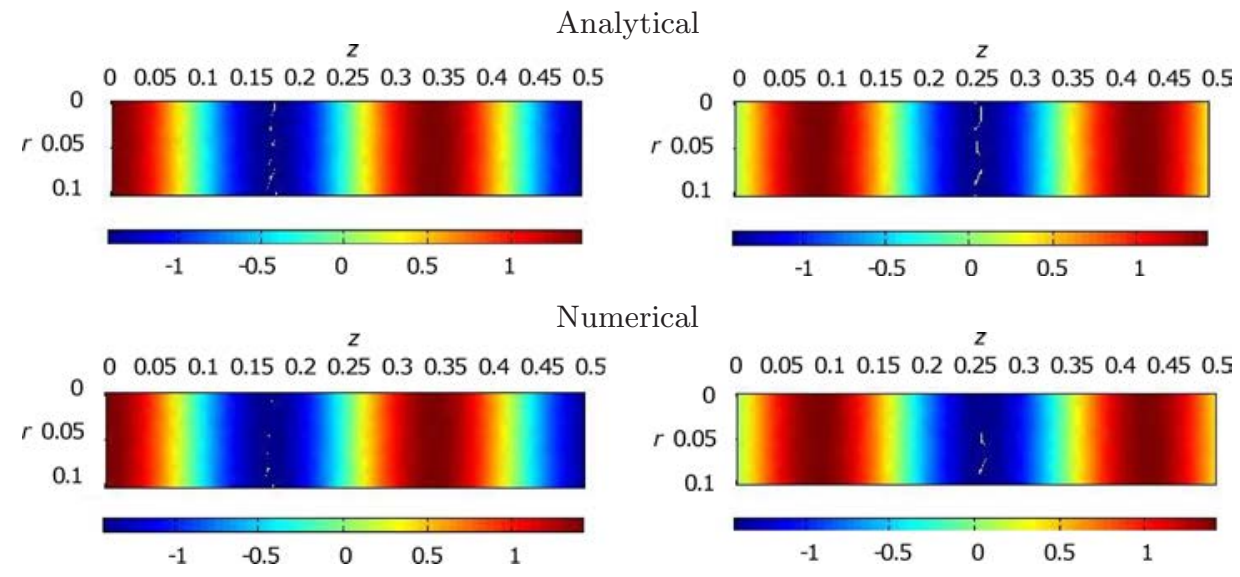

Fig. 5. Real part (left) and imaginary part (right) of the acoustic pressure in Pa inside of the studied wall duct at $f=1000 \mathrm{~Hz}$ and for $M_{0}=0$ when imposing the $(0,0)$ mode.
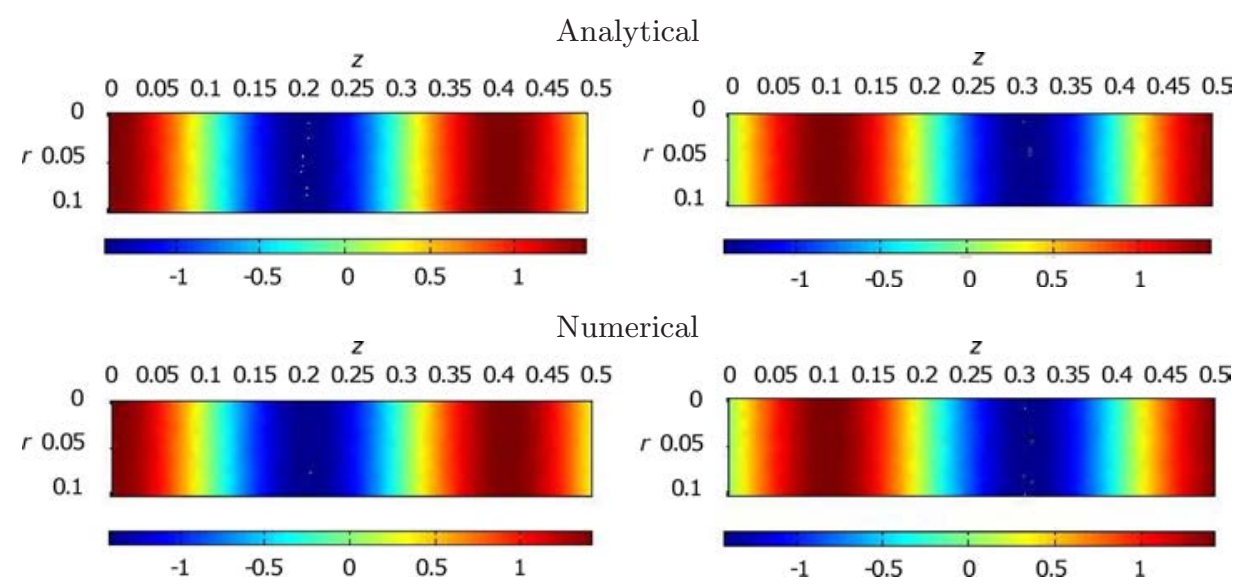

Fig. 6. Real part (left) and imaginary part (right) of the acoustic pressure in $\mathrm{Pa}$ inside of the studied wall duct at $f=1000 \mathrm{~Hz}$ and for $M_{0}=0.2$ when imposing the $(0,0)$ mode. 


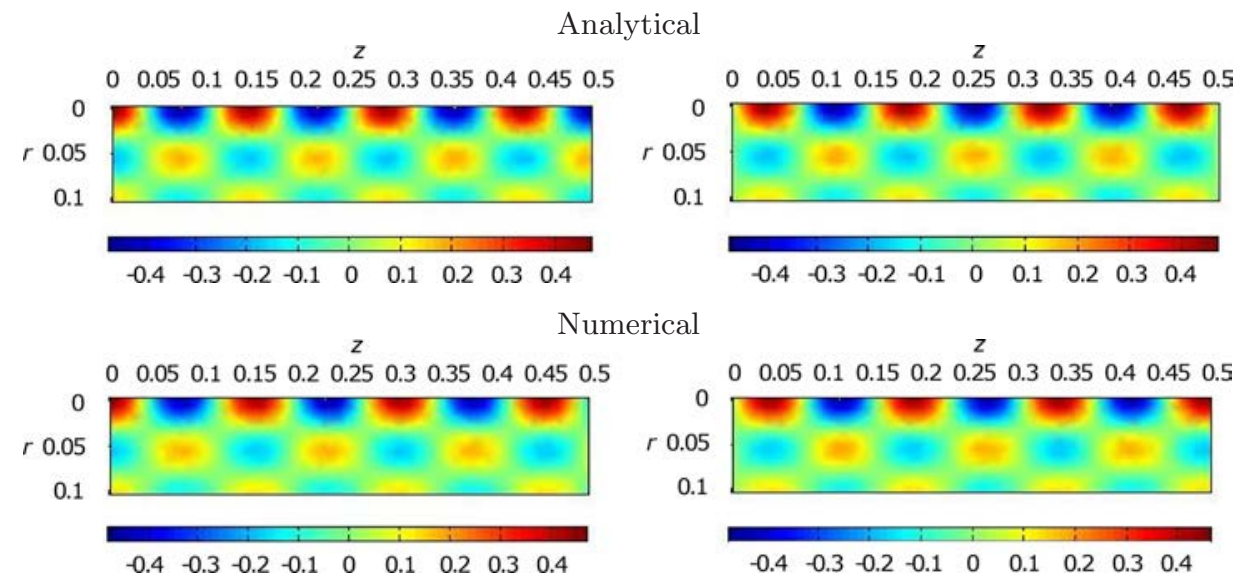

Fig. 7. Real part (left) and imaginary part (right) of the acoustic pressure in Pa inside of the studied wall duct at $f=5000 \mathrm{~Hz}$ and for $M_{0}=0.2$ when imposing the $(0,2)$ mode.

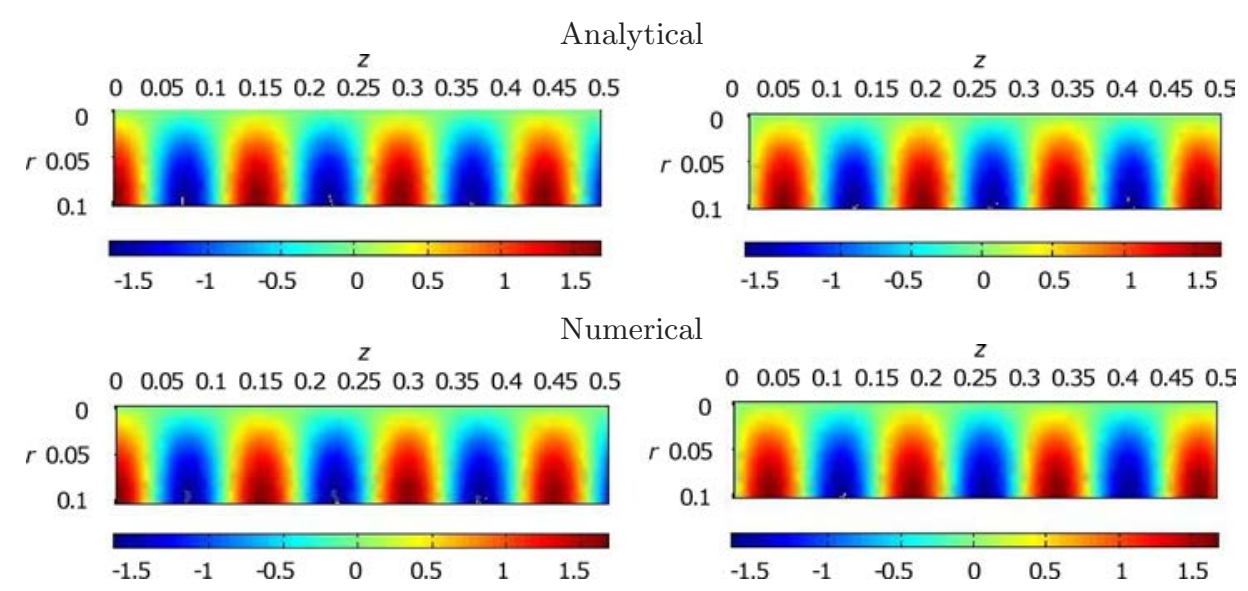

Fig. 8. Real part (left) and imaginary part (right) of the acoustic pressure in Pa inside of the studied wall duct at $f=3000 \mathrm{~Hz}$ and for $M_{0}=0.2$ when imposing the $(1,0)$ mode.

Figures 4, 5, and 6 show a good agreement between the numerical and analytical solutions. These figures show well the propagation of the plane wave inside the duct, as well as the flow effect. The increase of the Mach number in the same $z$ direction generates a shift of the wave in the same direction: the maximum of the real part of the acoustic pressure $(1.5 \mathrm{~Pa})$ located at $z=0.35 \mathrm{~m}$ in the no flow case is shifted to $z=0.41 \mathrm{~m}$ when $M_{0}=0.2$ and to $z=0.27$ for $M_{0}=-0.2$. The same remark is observed for the imaginary part: the maximum of the imaginary part located at $z=0.44 \mathrm{~m}$ for the case of no flow is shifted to $z=0.5$ for $M_{0}=0.2$ and to $z=0.35 \mathrm{~m}$ for $M_{0}=-0.2$.

As a second step, we impose a radial mode on the left boundary of the studied rigid wall duct. Figure 7 presents a comparison between the numerical and analytical solutions of the inside acoustic pressure when imposing $(0,2)$ mode at $f=5000 \mathrm{~Hz}$ and for $M_{0}=0.2$. Like in the $(0,0)$ mode case, a good agreement between the numerical and analytical results is observed. Figure 8 presents the acoustic pressure field inside the studied wall duct when imposing the azimuthal mode
$(1,0)$ at $f=3000 \mathrm{~Hz}$ and with $M_{0}=0.2$. These figures show a good agreement between the numerical and analytical results.

\subsection{Case No. 2: Cylindrical lined duct}

The studied duct in this case has the same geometrical characteristics as this of the first case but totally lined by a liner with a constant acoustic impedance $Z=\rho_{0} c_{0}(1+i)$. We begin by imposing the $(0,0)$ mode at the left boundary of the duct. Figures 9, 10, and 11 present a comparison between the analytical and numerical results of the inside acoustic pressure fields at $f=1000 \mathrm{~Hz}$ respectively for $M_{0}=-0.2,0$ and 0.2 . Figure 12 shows a comparison between the numerical and analytical pressure fields inside the studied lined duct at $f=7000 \mathrm{~Hz}$ with $M_{0}=0.2$ by imposing the $(2,1)$ mode at the duct entry. Figure 13 presents a comparison between the numerical and analytical pressure fields inside the studied lined duct at $f=7000 \mathrm{~Hz}$ with $M_{0}=0.2$ by imposing the $(0,2)$ mode at the duct entry. 
Figures 9 to 13 reveal that even if the duct edges are lined, the developed numerical method gives good results in accordance with the analytical results. These results show the effect of the liner on the propaga- tion wave which is attenuated when propagating inside the duct. It is also observed that if the Mach number increases the attenuation of the wave decreases.

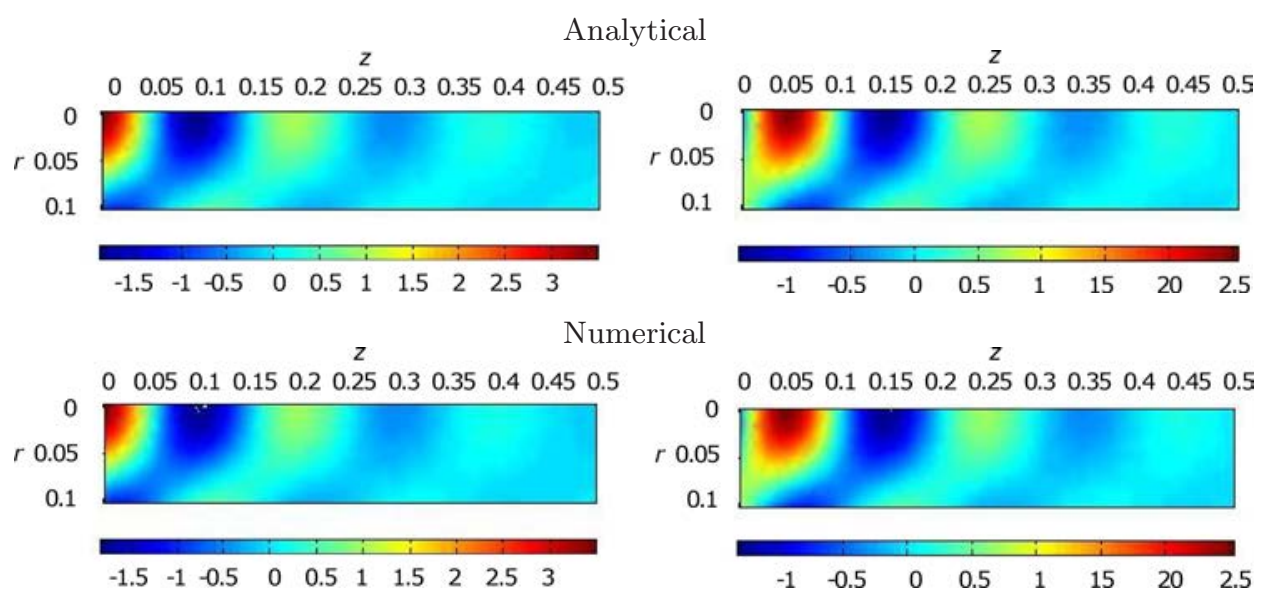

Fig. 9. Real part (left) and imaginary part (right) of the acoustic pressure in Pa inside of the studied lined duct at $f=2000 \mathrm{~Hz}$ and for $M_{0}=-0.2$ when imposing the $(0,0)$ mode.

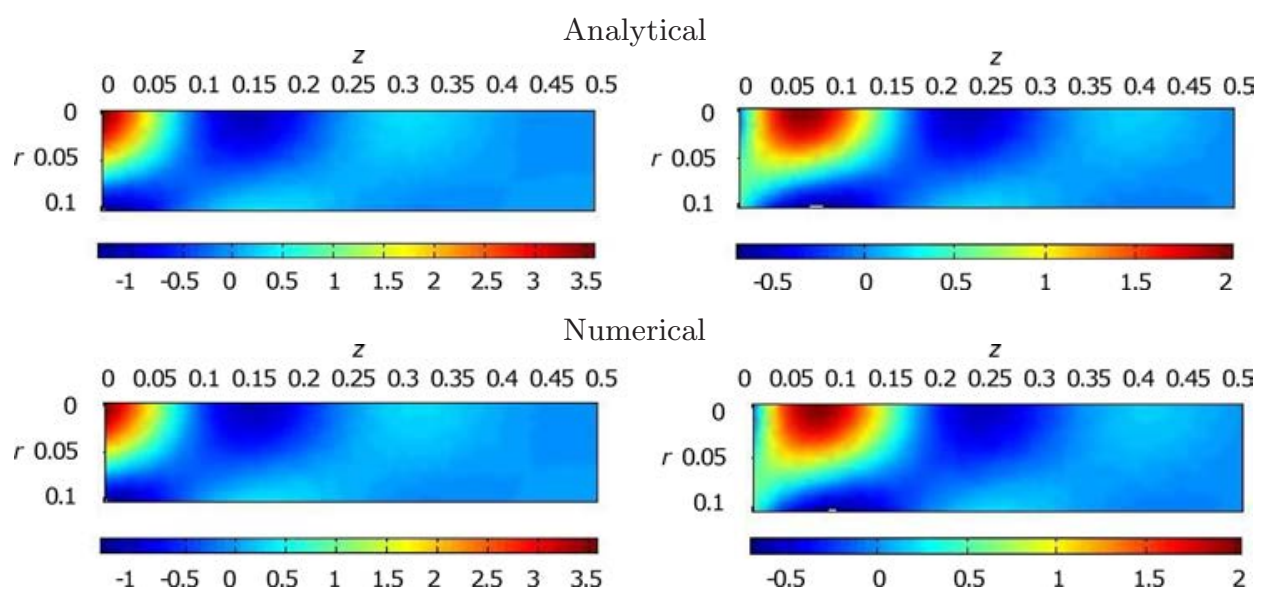

Fig. 10. Real part (left) and imaginary part (right) of the acoustic pressure in Pa inside of the studied lined duct at $f=2000 \mathrm{~Hz}$ and for $M_{0}=0$ when imposing the $(0,0)$ mode.
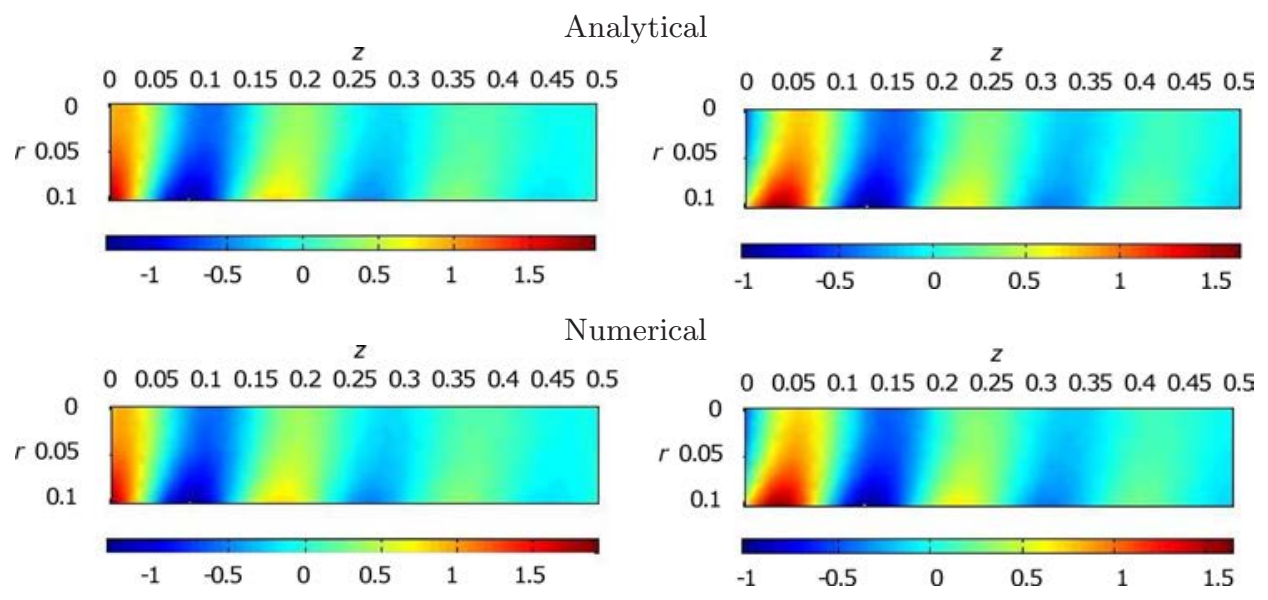

Fig. 11. Real part (left) and imaginary part (right) of the acoustic pressure in Pa inside of the studied lined duct at $f=2000 \mathrm{~Hz}$ and for $M_{0}=0.2$ when imposing the $(0,0)$ mode. 


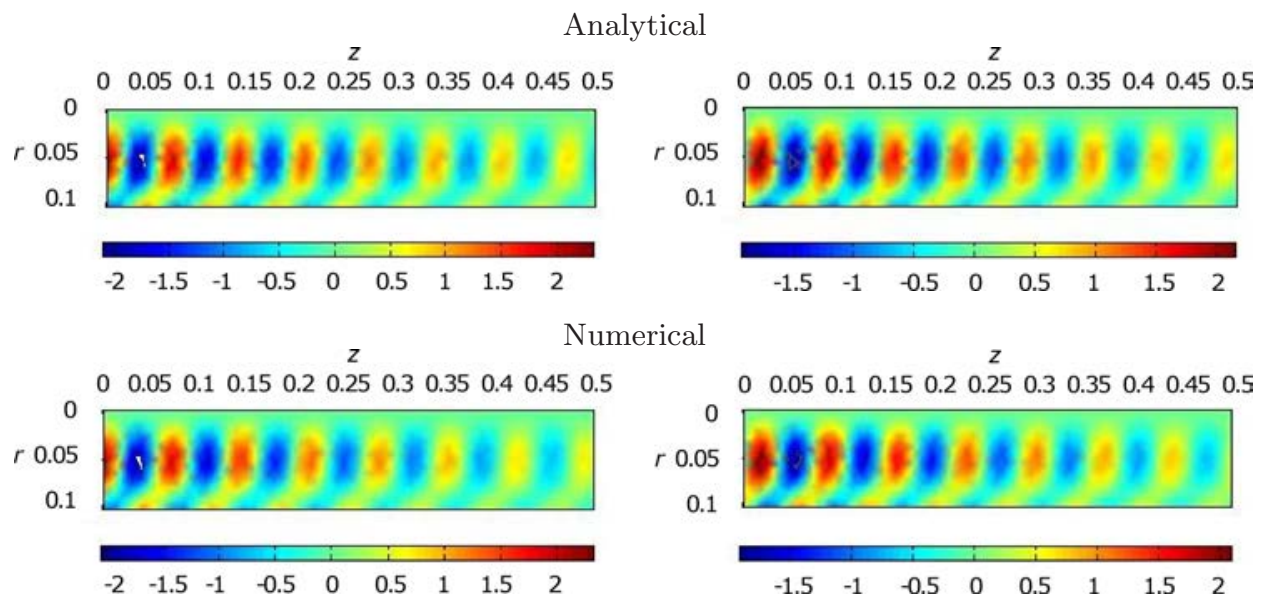

Fig. 12. Real part (left) and imaginary part (right) of the acoustic pressure in Pa inside of the studied lined duct at $f=7000 \mathrm{~Hz}$ and for $M_{0}=0.2$ when imposing the $(2,1)$ mode.

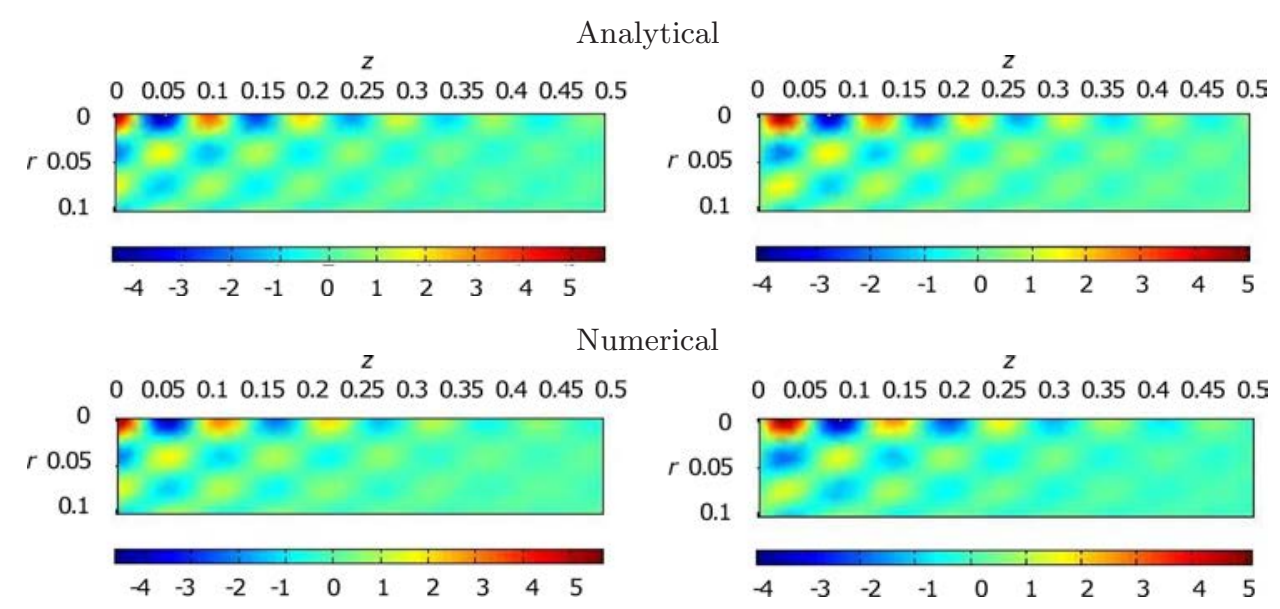

Fig. 13. Real part (left) and imaginary part (right) of the acoustic pressure in Pa inside of the studied lined duct at $f=7000 \mathrm{~Hz}$ and for $M_{0}=0.2$ when imposing the $(0,2)$ mode.

From these results we conclude that whatever the frequency, the imposed mode, and the edge boundaries, the proposed numerical method gives good results in accordance with the analytical ones.

\section{Conclusion}

In this study, a numerical method for the modeling of the acoustic pressure inside an axisymmetric lined duct in the presence of a flow was developed and presented. This method is based on the resolution of the convected Helmholtz equation with boundaries conditions. The proposed method gives good results as compared with the analytical ones. By varying the flow velocity, its effect was evaluated: the increase of the flow decreases the attenuation of the acoustic pressure inside the duct. Several ameliorations can be added to the proposed finite element method to model threedimensional flow ducts.

\section{Acknowledgment}

This work was carried out within the framework of the Tunisian-French research project DGRSRT/CNRS 09/R 11-43 dedicated to modelling of vibro-acoustic problems.

The authors would like to thank Mr H. BEJAOUI from the Faculty of Sciences of Sfax (Tunisia) for his help with English.

\section{References}

1. Bi W.P., Pagneux V., Lafarge D., Aurégan Y. (2003), Sound propagation in non-uniform lined duct by the multimodal method, Proceedings of 10th International Conference on Sound and Vibration, pp. 32293236, Stockholm.

2. Bi W.P., Pagneux V., Lafarge D., Aurégan Y. (2006), Modelling of sound propagation in non-uniform 
lined duct using a Multi-Modal Propagation Method, Journal of Sound and Vibration, 289, 1091-1111.

3. Chang Y.C., Chiu M.C. (2010), Optimization of multi-chamber mufflers with reverse - flow ducts by algorithm of simulated annealing, Archives of Acoustics, 35, 1, 13-33.

4. Chiu M.C. (2009), Simulated annealing optimization on multi-chamber mufflers hybridized with perforated plug - inlet space constraints, Archives of Acoustics, 34, 3, 305-543.

5. Dhatt G., Touzot G. (1989), Presentation of the finite elements method, Maloine S.A. Editeur, Paris.

6. Dougherty R.P. (1997), A wave-splitting technique for nacelle acoustic propagation, AIAA Paper 97-1652.

7. Dougherty R.P. (1999), A parabolic approximation for flow effects on sound propagation in non-uniform sofwall ducts, AIAA Paper 99-1822.

8. Dykas S., Wroblewski W., Rulik S., ChmielNIAK T. (2010), Numerical method for modeling of acoustic waves propagation, Archives of Acoustics, 35, $1,35-48$.

9. Elnady T. (2004), Modeling and characterization of perforates in lined ducts and mufflers, Ph.D. Thesis, The Royal Institute of Technology.

10. Jones M.G., Watson W.R., Parrott T.L. (2005), Benchmark data for evaluation of aeroacoustic propagation codes with grazing flow, AIAA paper 2005-2853.

11. LAN J.H., Bread C. (2005), Validation of $3 D$ acoustic propagation code with analytical and experimental results, AIAA Paper 2005-2901.

12. LAPKA W. (2009), Insertion Loss of Spiral Ducts Measurements and Computations, Archives of Acoustics, 34, 4, 537-545.

13. Leroux M., Job S., Aurégan Y., Pagneux V. (2003), Acoustical propagation in lined duct with flow. Numerical simulations and measurements, Proceedings of 10th International Conference on Sound and Vibration, pp. 3255-3262, Stockholm.

14. LeSUEUR L. (1988), Rayonnement acoustique des structures: Vibroacoustique et Interactions Fluide structure, Editions Eyrolles, Paris.

15. Lin W.H. (1998), A least-squares spectral element method for sound propagation in acoustic ducts, Journal of the Acoustical Society of America, 104, 5, 31113114 .

16. McAlpine A., Fisher M.J. (2003), On the prediction of "buzz-saw" noise in acoustically lined aero-engine inlet ducts, Journal of Sound and Vibration, 265, 175200 .

17. Meissner M. (2010), Effect of cross-sectional area discontinuities in closed hard-walled ducts on frequency of longitudinal modes, Archives of Acoustics, 35, 3, 421435.

18. Nark D.M., Farassat F., Pope D.S., Vatsa V. (2003), The development of the ducted fan noise propagation and radiation code CDUCT-LaRc, AIAA Paper 2003-3242.

19. NARK D.M., WATson W.R., Jones M.G. (2005), An investigation of two acoustic propagation codes for three dimensional geometries, AIAA Paper 2005-3022.

20. NARK D.M., WATson W., Jones M. (2006), Further Investigation of two acoustic propagation codes for three-dimensional geometries, AIAA Paper 2006-2586.

21. ÖZyÖrük Y., Alpman E., Ahuja V., Long L.N. (2004), Frequency domain prediction of turbofan noise radiation, Journal of Sound and Vibration, 270, 933950.

22. ÖZYÖRÜK Y., LONG L.N. (1996), Computational of sound radiating from engine inlets, AIAA Journal, $\mathbf{3 4}$ $5,894-901$.

23. Peat K.S., Rathi K.L. (1995), Finite element analysis of the convected acoustic wave motion in dissipative silencers, Journal of Sound and Vibration, 184, 3, 529545 .

24. Reddy J.N. (1993), An Introduction to Finite Element Methods, Second Edition, McGraw Hill Inc. New York.

25. Reichert R.S., Biringen S. (1997), Time domain simulation of acoustic propagation in a lined duct, AIAA Paper 97-1650.

26. Sbardella L., Tester B.J., Imergun M. (2001), A time-domain method for prediction of sound attenuation in lined duct. Journal of Sound and Vibration, 239, 3, 379-396.

27. Stanescu D., Ait Ali Yahia A., Habashi W.G., RoBICHAUD B. (1999), Multidomain spectral computation of sound radiation from ducted fans, AIAA Journal, 37, 3, 296-302.

28. Taktak M., Jrad H., Karra C., Bentahar M., HADDAR M. (2011), Numerical modeling of the acoustic propagation in a three dimensional wave guides in the presence of flow, Acta Acustica united with Acustica, 97, 3, 453-465.

29. Watson W.R., Jones M.G. (2006), Comparison of a convected Helmholtz and Euler model for impedance eduction in flow, AIAA Paper 2006-2643.

30. Watson W.R., Jones M.G., Parrott T.L. (2005), A quasi-3D Theory for impedance eduction in uniform flows, AIAA Paper 2005-2848.

31. Watson W.R., Jones M.G., Tanner S.E., Parrott T.L. (1996), A finite element propagation model for extracting normal incidence impedance in nonprogressive acoustic wave fields, Journal of Computational Physics, 125, 1, 177-186 